Sharlene Murdoch, Muheiddine Seoud, Renate Kircheisen, Batool Mazhar, and Rima Slim

\title{
Detailed gene and allele content analysis of three homozygous KIR haplotypes
}

Published in:

Tissue Antigens 68 (1):72-77, 2006

This article may be used for non-commercial purposes in accordance with Wiley Terms and Conditions for Self-Archiving:

http://olabout.wiley.com/WileyCDA/Section/id-820227.html\#terms

doi: 10.1111/j.1399-0039.2006.00606.x

http://onlinelibrary.wiley.com/doi/10.1111/j.1399-

0039.2006.00606.x/abstract 


\section{Full Title:}

Detailed gene and allele content analysis of three homozygous KIR haplotypes

Short Title:

Analysis of three homozygous KIR haplotypes

Sharlene Murdoch ${ }^{1,2}$, Muheiddine Seoud ${ }^{3}$, Renate Kircheisen ${ }^{4}$, Batool Mazhar ${ }^{5}$, Rima Slim ${ }^{1,2}$

${ }^{1}$ Departments of Human Genetics and ${ }^{2}$ Obstetrics and Gynaecology, McGill University Health Centre, Montreal H3G 1A4, Canada

${ }^{3}$ Dept of Obstetrics \& Gynaecology, American University of Beirut, P.O. Box 11-236, Lebanon

${ }^{4}$ Institut für Klinische Genetik, Mainz 55131, Germany

${ }^{5}$ Dept of Obstetrics and Gynaecology, Maternal \& Child Health Centre Unit-II, Pakistan Institute of Medical Sciences, Islamabad, Pakistan

Correspondence:

Rima Slim

Montreal General Hospital Research Institute, room L12-132

1650 Cedar Avenue

H3G 1A4, Montreal, Canada

Tel: $\quad$ (514) 934-1934 ext 44550

Fax: $\quad$ (514) 9348261

e-mail : $\quad$ rima.slim@muhc.mcgill.ca 


\begin{abstract}
The Killer Immunoglobulin-like Receptors are a highly polymorphic family of receptors encoded by fifteen genes clustered on 19q13.4. Due to the complexity of the genetic analysis of the KIR cluster much of the data regarding KIR sequences and alleles has been generated by cDNA typing and partial sequencing. Here we report the genomic sequencing of the KIR genes in individuals with three different haplotypes homozygous by descent. We provide a detailed analysis of their haplotypes and identify new alleles for KIR3DL3 and KIR2DL1. The primers we describe will be a valuable tool for studying the involvement of the KIR genes in various human diseases.
\end{abstract}


The Killer Immunoglobulin-like Receptors (KIRs) make up a family of membrane bound receptors found on the surface of Natural Killer (NK) cells and some T-cells (1). The family is comprised of seventeen genes, fifteen of which are expressed and two are pseudogenes, arranged in a head to tail fashion over a $150 \mathrm{~kb}$ region on 19q13.4 (1). All seventeen genes are believed to have evolved from a single ancestral KIR gene after the divergence of the hominoid line from mice (2), as mice have only two KIR-like genes and instead rely on the Ly49 family of C-type lectin receptors for NK cell regulation (3). The KIR haplotypes have been repeatedly shuffled by reciprocal and non-reciprocal crossing over events and thus, aside from the four "framework" genes which are present in all individuals (KIR3DL3, KIR3DP1, KIR2DL4 and KIR3DL2), they are highly variable with respect to the number and types of genes they contain $(4,5)$. At the gene content level haplotypes can be divided into two broad groups: A and B (6). Though each group contains multiple subtypes, in general, the A haplotype contains fewer genes, most of which encode inhibitory receptors, while the B haplotype is longer and contains more activating receptors (7). To date over a hundred different haplotypes have been identified at the gene level in healthy subjects (8). To add further diversity to the haplotypes, each gene has multiple alleles, from three for the pseudogene KIR2DP1, up to twenty-two identified thus far for KIR2DL4 (http://www.ebi.ac.uk/ipd/kir/stats.html). As a result, complete homozygosity, with respect to gene content and alleles, for a given KIR haplotype is very rare. Despite the polygenicity and polymorphism of the KIR complex, there remains a high degree of homology -more than $90 \%$ (4)- between the intronic and exonic sequences of the different genes. This genetic complexity makes it very difficult to design unique genomic primers to PCR amplify and sequence the different exons 
directly. Consequently, much of what we know of KIR gene sequences comes from partial cDNA sequencing (6), with the exception of a recent extensive study of the KIR2DL4 gene (9).

KIR receptors bind HLA class I ligands and regulate NK cell function (1). Increasingly associations are being made between KIR haplotypes or specific genes and human diseases, such as rheumatoid arthritis (10), psoriasis (11), disease progression in HIV infection (12), hepatitis C (13), cervical cancer (14), pre-eclampsia and reproductive failure (15). However, due to the complexity of the genetic analysis of the KIR genes, no causative gene defect has been found in any of the KIRs for these diseases. Recurrent hydatidiform moles (HMs) is an autosomal recessive disease caused by mutations in NALP7 (16), a gene located $50 \mathrm{~kb}$ distal to the KIR cluster. Several patients with defects in NALP7 are homozygous over the entire KIR haplotype. In this study, we took advantage of this situation and sequenced the coding regions of most of the genes in three such patients, MoLb1-4, MoGe2-2, and MoPa61-3. Here we describe a detailed analysis of the gene and allelic content of their three haplotypes. The primers we provide will facilitate the sequencing of the KIR genes and contribute to a better understanding of their roles in various human diseases.

The predicted gene structure of each KIR (http://genome.ucsc.edu/cgi-bin/hgGateway) was used to design specific genomic primers to PCR-amplify all exons. The primers and conditions used are provided in Table 1. Amplicons were sequenced directly or after cloning and the sequences were compared to the KIR database 
(http://www.ebi.ac.uk/ipd/kir/) to determine the allele names. The haplotypes of the patients are presented in Figure 1. The gene content of each haplotype was also confirmed using previously published KIR profiling primers (6). Patient MoPa61-3 has a haplotype corresponding to an A group, where MoLb1-4 and MoGe2-2 have haplotypes consistent with a B group. For two genes, KIR2DS2 and KIR2DS3, present only in MoGe2-2, we were unable to design specific primers to amplify their exons due to the lack of genomic sequences containing these genes. However, when we cloned the other genes, some of the clones contained sequences corresponding to KIR2DS2 and KIR2DS3 and we were able to confirm their presence, but could not determine their alleles. For most genes, the patients had known alleles (Figure 1), with the exception of KIR2DL1 in MoGe2-2 and KIR3DL3 in MoLb1-4 and MoPa61-3 (Figure 2). The SNPs in MoGe2-2 are almost identical to allele *00401 of KIR2DL1, but differ by one SNP: a C at position 796 leading to a non-synonymous polymorphism that results in a C245R change (IPD KIR2DL1 alignment release 1.1.1 http://www3.ebi.ac.uk/Services/ipd/kir/cgibin/align.cgi) in the transmembrane domain (Figure 2a). This change is seen in six other alleles of this gene but its presence in conjunction with the other SNPs observed for this patient defines a new allele (Genbank accession nos. DQ371485-92). Similarly, for KIR3DL3 MoPa61-3 differs from the reference allele $* 001$ by just one SNP in the transmembrane domain at nucleotide position 961 (Genbank accession nos. DQ37150108). This allele is identical to the KIRC1TM3 varient identified by Trundley et al. in a recent study (17). MoLb1-4, however, has three SNPs in KIR3DL3 that do not correspond to any known allele in the databases (Figure 2b). The SNPs at positions 621 and 1074 are synonymous, but the $\mathrm{T}$ at 447 leads to an amino acid substitution, R128S, in 
the second immunoglobulin binding domain. Another SNP, an $\mathrm{A}>\mathrm{C}$ at 869 , also leads to an amino acid change, N269T (IPD KIR3DL3 alignment release 1.1.1) in the third immunoglobulin binding domain. This change has been observed in two variants (variants 2 and 5) that are not found in the KIR sequence database but are found in GenBank (accession nos. AJ938063.1 and AJ938066.1, respectively). These variants do not contain the other SNPs observed in MoLb1-4, again making this combination of SNPs unique (Genbank accession nos. DQ371493-1500).

We report a detailed description of three homozygous KIR haplotypes in women with familial recurrent HMs. To our knowledge, this is the first report of complete homozygosity at the gene and allele levels over the entire KIR cluster. Homozygosity at the gene content level for the A haplotype has been associated with an increased risk for pre-eclampsia when the foetus carries the HLA-C2 group (15), and having a KIR repertoire with a limited number of inhibitory genes has been linked to an increased risk of spontaneous abortions (18). Both pre-eclamptic features and spontaneous abortions are part of the clinical spectrum observed in patients with recurrent hydatidiform moles (19) (and published data). Although the molar phenotype in these patients is caused by mutations in NALP7, a gene known to play a role in inflammation (20), the KIR receptors are also part of the inflammatory response as they modulate the secretion of various cytokines by NK cells. This suggests that, while homozygosity for the KIR genes does not play a causal role in recurrent molar pregnancies in these patients, its role in modulating the disease phenotype and its subsequent sequelae cannot be excluded at 
present time. The analysis of the KIR haplotypes in additional patients with HMs caused by mutations in $N A L P 7$ will answer this question.

A major international effort is underway to type and sequence all the KIR genes. Because of their high homology and diversity, determining the gene and allele content of individuals with heterozygous haplotypes requires the typing of their parents or siblings to establish the phase. The identification of subjects homozygous over the KIR cluster provided an easier mean to fully sequence the various KIR exons, splice sites, flanking introns, and regulatory elements and hence will facilitate the screening of these alleles for mutations in patients with various diseases believed to be caused by the KIR genes.

\section{Acknowledgements}

We would like to thank the members of the families for their participation in this study. R.S. is supported by the Fonds de la Recherche en Santé du Québec, and by an operating and an international development grants from the Canadian Institutes of Health Research.

Competing interests statement The authors declare that they have no competing financial interests. 


\section{References}

1. Moretta L, Moretta A. Killer immunoglobulin-like receptors. Curr Opin Immunol 2004; 16 5: 626-33.

2. Rajalingam R, Parham P, Abi-Rached L. Domain shuffling has been the main mechanism forming new hominoid killer cell Ig-like receptors. J Immunol 2004; 172 1: 356-69.

3. $\quad$ Lanier LL. NK cell receptors. Annu Rev Immunol 1998; 16: 359-93.

4. Uhrberg M. The KIR gene family: life in the fast lane of evolution. Eur $J$ Immunol 2005; 35 1: 10-5.

5. Martin MP, Bashirova A, Traherne J, Trowsdale J, Carrington M. Cutting edge: expansion of the KIR locus by unequal crossing over. J Immunol 2003; 171 5: 2192-5.

6. Uhrberg M, Valiante NM, Shum BP, et al. Human diversity in killer cell inhibitory receptor genes. Immunity 1997; 7 6: 753-63.

7. Martin AM, Kulski JK, Gaudieri S, et al. Comparative genomic analysis, diversity and evolution of two KIR haplotypes A and B. Gene 2004; 335: 121-31.

8. Carrington and Norman. The KIR gene cluster. (http://www.ncbi.nlm.nih.gov/books/), 2003.

9. Gedil MA, Steiner NK, Hurley CK. Genomic characterization of KIR2DL4 in families and unrelated individuals reveals extensive diversity in exon and intron sequences including a common frameshift variation occurring in several alleles. Tissue Antigens 2005; 65 5: 402-18.

10. Yen JH, Moore BE, Nakajima T, et al. Major histocompatibility complex class Irecognizing receptors are disease risk genes in rheumatoid arthritis. J Exp Med 2001; 193 10: 1159-67.

11. Luszczek W, Manczak M, Cislo M, et al. Gene for the activating natural killer cell receptor, KIR2DS1, is associated with susceptibility to psoriasis vulgaris. Hum Immunol 2004; 65 7: 758-66.

12. Martin MP, Gao X, Lee JH, et al. Epistatic interaction between KIR3DS1 and HLA-B delays the progression to AIDS. Nat Genet 2002; 31 4: 429-34.

13. Khakoo SI, Thio CL, Martin MP, et al. HLA and NK cell inhibitory receptor genes in resolving hepatitis C virus infection. Science 2004; 305 5685: 872-4.

14. Carrington M, Wang S, Martin MP, et al. Hierarchy of resistance to cervical neoplasia mediated by combinations of killer immunoglobulin-like receptor and human leukocyte antigen loci. J Exp Med 2005; 201 7: 1069-75.

15. Hiby SE, Walker JJ, O'Shaughnessy K M, et al. Combinations of maternal KIR and fetal HLA-C genes influence the risk of preeclampsia and reproductive success. J Exp Med 2004; 200 8: 957-65.

16. Murdoch S, Djuric U, Mazhar B, et al. Mutations in NALP7, a maternal effect gene, result in recurrent hydatidiform moles and reproductive wastage in humans. Nature Genetics; (in press).

17. Trundley AE, Hiby SE, Chang C, Sharkey AM, Santourlidis S, Uhrberg M, Trowsdale J, Moffett A. Molecular characterization of KIR3DL3. Immunogenetics 2006;57: 904-16. 
18. Varla-Leftherioti M, Spyropoulou-Vlachou M, Niokou D, et al. Natural killer (NK) cell receptors' repertoire in couples with recurrent spontaneous abortions. Am J Reprod Immunol 2003; 49 3: 183-91.

19. Seoud M, Khalil A, Frangieh A, Zahed L, Azar G, Nuwayri-Salti N. Recurrent molar pregnancies in a family with extensive intermarriage: report of a family and review of the literature. Obstet Gynecol 1995; 864 Pt 2: 692-5.

20. Kinoshita T, Wang Y, Hasegawa M, Imamura R, Suda T. PYPAF3, a PYRINcontaining APAF-1-like Protein, Is a Feedback Regulator of Caspase-1-dependent Interleukin-1 \{beta\} Secretion. J Biol Chem 2005; 280 23: 21720-5.

21. Hsu KC, Chida S, Geraghty DE, Dupont B. The killer cell immunoglobulin-like receptor (KIR) genomic region: gene-order, haplotypes and allelic polymorphism. Immunol Rev 2002; 190: 40-52. 
Figure 1

\begin{tabular}{|c|c|c|c|c|c|c|c|c|c|c|c|c|c|c|}
\hline & 3DL3 & 2DS2 & $\begin{array}{l}2 \mathrm{DL} 2 \\
2 \mathrm{DL} 3 \\
\end{array}$ & 2DL5B & $\begin{array}{l}2 \mathrm{DS} 3 \\
2 \mathrm{DS} 5 \\
\end{array}$ & 2DL1 & $2 \mathrm{DL} 4$ & $\begin{array}{l}\text { 3DS1 } \\
\text { 3DL1 }\end{array}$ & 2DL5A & $2 \mathrm{DS} 3$ & $2 \mathrm{DS} 5$ & $2 \mathrm{DS} 1$ & $2 \mathrm{DS} 4$ & 3DL2 \\
\hline MoGe2-2 & ${ }^{*} 003$ & & L2*001 & *002 & S3 & New & ${ }^{\circ} 00801$ & L1 ${ }^{*} 00101$ & & & & & ${ }^{0} 003$ & ‘001 \\
\hline MoLb1-4 & New & & L3*002 & & & ${ }^{*} 002$ & ${ }^{*} 005$ & $S 1 * 013$ & ${ }^{*} 001$ & & ${ }^{*} 002$ & ${ }^{*} 002$ & & ${ }^{*} 007$ \\
\hline loPa61-3 & New & & L3*001 & & & ${ }^{*} 00302$ & ${ }^{*} 00801$ & L1“00101 & & & & & ${ }^{\circ} 003$ & ‘001 \\
\hline
\end{tabular}

Figure 2

a

2DL1

\begin{tabular}{|c|c|c|c|c|c|c|c|c|c|c|c|c|c|c|c|}
\hline \multirow[b]{2}{*}{ Nucleotide } & \multirow{2}{*}{\begin{tabular}{|c|} 
Exon 1 \\
13 \\
\end{tabular}} & \multicolumn{2}{|c|}{ Exon 4} & \multicolumn{6}{|c|}{ Exon 5} & \multicolumn{2}{|c|}{ Exon 6} & \multicolumn{2}{|c|}{ Exon 7} & \multirow{2}{*}{\begin{tabular}{c|} 
Exon 8 \\
849 \\
\end{tabular}} & \multirow{2}{*}{\begin{tabular}{|c|} 
Exon 9 \\
997 \\
\end{tabular}} \\
\hline & & 110 & 144 & 404 & 523 & 549 & 550 & 576 & 608 & 709 & 714 & 796 & 813 & & \\
\hline Reference & $G$ & $c$ & A & $C$ & $c$ & $T$ & $G$ & $C$ & A & A & $C$ & $c$ & A & A & A \\
\hline MoGe2-2 & - & $G$ & - & - & $\mathrm{A}$ & $C$ & $\mathrm{~A}$ & $\mathrm{~T}$ & $G$ & G & - & - & - & - & - \\
\hline MoLb1-4 & $\mathrm{T}$ & - & - & - & - & - & - & - & - & - & - & - & - & - & - \\
\hline $\mathrm{MoPa} 61-3$ & - & G & G & $\mathrm{T}$ & - & - & - & - & - & - & - & - & - & - & - \\
\hline
\end{tabular}

b

3DL3

\begin{tabular}{|c|c|c|c|c|c|c|c|c|c|c|c|c|}
\hline \multirow[b]{2}{*}{ Nucleotide } & \multicolumn{3}{|c|}{ Exon 3} & \multicolumn{4}{|c|}{ Exon 4} & \multicolumn{2}{|c|}{ Exon 5} & \multicolumn{2}{|c|}{ Exon 7} & \multirow{2}{*}{$\begin{array}{c}\text { Exon } 8 \\
1074 \\
\end{array}$} \\
\hline & 155 & 354 & 408 & 447 & 502 & 621 & 777 & 804 & 869 & 961 & 971 & \\
\hline Reference & $G$ & A & $G$ & $G$ & A & $G$ & A & $G$ & $A$ & $A$ & $T$ & A \\
\hline MoGe2-2 & - & - & - & - & - & - & - & - & - & $T$ & $C$ & - \\
\hline MoLb1-4 & - & - & - & $\mathrm{T}$ & G & A & - & - & $C$ & - & - & G \\
\hline $\mathrm{MoPa61-3}$ & - & - & - & - & - & - & - & - & - & $C$ & - & - \\
\hline
\end{tabular}




\begin{tabular}{|c|c|c|c|c|c|c|c|c|c|c|c|c|c|c|c|c|c|c|c|c|}
\hline Gene & \multicolumn{2}{|c|}{ Exon } & Sequence & $\mathrm{T}^{\circ} \mathrm{C}$ & bp & Notes & Gene & Exo & & Sequence & $T^{\circ} \mathrm{C}$ & bp & Notes & Gene & \multicolumn{2}{|c|}{ Exon } & Sequence & $T^{\circ} \mathrm{C}$ & bp & Notes \\
\hline \multirow[t]{14}{*}{ 2DL1 } & \multirow{2}{*}{1} & $\mathrm{~F}$ & GTTCGGGAGGTTGGATCTCAGACG & \multirow{2}{*}{68} & \multirow{2}{*}{536} & \multirow{2}{*}{1} & \multirow[t]{14}{*}{ 2DL5AB } & \multirow{2}{*}{1} & $\mathrm{~F}$ & CCCAAGCCCATATCTCCATCCTAG & \multirow{2}{*}{60} & \multirow{2}{*}{760} & \multirow{2}{*}{2} & \multirow{2}{*}{\multicolumn{2}{|c|}{\begin{tabular}{|l|l} 
3DL3 & \\
\end{tabular}}} & $\mathrm{F}$ & GAACAGCCTGGGGAAAATAGCACA & \multirow{2}{*}{68} & \multirow{2}{*}{706} & \\
\hline & & $\mathrm{R}$ & TCCAGGCCCAGATCTCCACTACAG & & & & & & $\mathrm{R}$ & GTCTTTCACGTTAGCACAGATTTT & & & & & & $\mathrm{R}$ & AGGCCCGTATCTCAATTCCAGGTC & & & \\
\hline & 2 & $\mathrm{~F}$ & TATGGACCTGGAGTGGAGATAAGG & 68 & 815 & & & & $\mathrm{~F}$ & GTGGGGATAGGGGCCTGGGGTGCGG & 71 & 290 & & & & $\mathrm{~F}$ & ACGGGCCTGGAGGTGGAGATACAG & 68 & 91 & \\
\hline & & $\mathrm{R}$ & CAGCCGATGCCTGAACGAAAAT & 00 & (1) & & & & $\mathrm{R}$ & GCTGGGGAGGGCAAGGTCGGAACT & DIt & 290 & & & & $\mathrm{R}$ & TCCCCCTGGGTCCCCACAGACT & 100 & 451 & \\
\hline & 4 & $\mathrm{~F}$ & GTTCCTCTTCCACCCCCACATAGA & 71 & 719 & & & 3 & $\mathrm{~F}$ & CAGTTGTGTATTGTGGTTCACACA & 60 & 740 & & & 3 & $\mathrm{~F}$ & TGGGCACCCAGGTGTGGTAG & 77 & 08 & \\
\hline & & $\mathrm{R}$ & GCACAGACCTCACCAAGTCAGTC & 11 & 142 & & & & $\mathrm{R}$ & ATCCCCATTGACACCAAATACAGT & 60 & 140 & & & 3 & $\mathrm{R}$ & ATACAGTCTGGGCCTGGATGATTG & 17 & 508 & \\
\hline & 5 & $\mathrm{~F}$ & ACATGAAGAGCGATGGGGTAGAGG & 71 & 603 & 23 & & 5 & $\mathrm{~F}$ & CAGGGAGAGTCGGGGGTGGAGGGT & 60 & 955 & & & 4 & $\mathrm{~F}$ & TGCAACAGGGGTTATGGGCACAAA & 77 & 487 & \\
\hline & & $\mathrm{R}$ & GGGTTTGGAGGTGCCCTGTCG & (1) & . & 2,0 & & & $\mathrm{R}$ & CTCAAAGCACCGAGATTATAACTG & 100 & ovo & & & 7 & $\mathrm{R}$ & CCAGCAAGGGTGAGAGGCAGGTCT & די & 701 & \\
\hline & 6 & $\mathrm{~F}$ & GAGAGTGTTGGCCATGAACCATC & 66 & 442 & 23 & & & $\mathrm{~F}$ & CTGATTGCAGGTTCTTGGCACG & 60 & 600 & & & 5 & $\mathrm{~F}$ & AGAGATGGGGGTGGAGGGTGAG & 77 & 538 & \\
\hline & 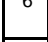 & $\mathrm{R}$ & CCTCCAGTTAGGAATGCAGGTAGA & 60 & 442 & 2,3 & & 6 & $\mathrm{R}$ & CACTGAGCCCTTTGCTGTCCTC & 100 & 600 & & & 5 & $\mathrm{R}$ & TGTGTTCTGTCTCGGCATCTGTCC & 17 & 330 & \\
\hline & 7 & $\mathrm{~F}$ & TCGTATCCTCAGCACGTTCTATGG & 68 & 511 & & & 7 & $\mathrm{~F}$ & TCTCATGGGGACAGCATTAATGTA & 60 & 525 & & & 7 & $\mathrm{~F}$ & CCCAAGAGGCCCAACCTCCCACCC & 77 & 681 & \\
\hline & $r$ & $\mathrm{R}$ & AGGGGACATGGGGATACAGTTCAG & 00 & דו गנ & & & 17 & $\mathrm{R}$ & CAGTCAGGAACACACACCAGTGTG & 100 & OLS & & & $t$ & $\mathrm{R}$ & ATCCATCCCATGTATAGCTCTGAGT & trit & 001 & \\
\hline & & $\mathrm{F}$ & GAGGGACCTCAGCCACCTATGG & & & & & & $F$ & AGAATGTCTGGGTCTGGCTGATGA & & & & & & $\mathrm{F}$ & AAGTGCCCTCCGAGCTGTTTTGAC & & & \\
\hline & $8-9$ & $R$ & TGTGAGGAAGATCGATGCCCTAAG & 66 & 573 & & & 8-9 & $\mathrm{R}$ & CACTGCTGACTGACAGAAGGCTGG & 68 & 910 & & & $8-9$ & $\mathrm{R}$ & TAGGCAAGAAAAGAGTCCCATTGA & 68 & 846 & \\
\hline $2 \mathrm{DL} 3 / 2$ & 1 & $\mathrm{~F}$ & CCCAGGTTCAAGCTATTCTGATGC & 71 & 744 & 4 & 2DS4 & & $\mathrm{F}$ & GTTTGGGAGGTTGGATCTAAGACA & 66 & 531 & & $2 \mathrm{DS} 1$ & 1 & $\mathrm{~F}$ & ACAGAGAGAGAGCAAACTCCAG & 03 & $77 ?$ & \\
\hline & & $\mathrm{R}$ & CCAGGCCCATGTCTCCACTACAAG & 71 & 744 & 4 & & 1 & $\mathrm{R}$ & CATCTCTAGGCCCAGATCTCCACT & 60 & 531 & & & & $\mathrm{R}$ & СACTCCCTTCCTCTATTCCCT & 100 & $10<$ & \\
\hline & 2 & $\mathrm{~F}$ & GGAGATATGGGCCTAGGAAGGAGA & 66 & 612 & 4 & & 2 & $\mathrm{~F}$ & GCCCGGATTGGCTATATGGGTCTA & 68 & 469 & & & 2 & $\mathrm{~F}$ & AGAGGAAGGGAGTGTGGGGTTG & 70 & 1403 & \\
\hline & & $\mathrm{R}$ & GAGAGGCCAGGGAGGCGAGGTC & 00 & 016 & 4 & & & $\mathrm{R}$ & TGAGAGGCCAGGGAGGCAAGGTC & 100 & 405 & & & 2 & $\mathrm{R}$ & CAGCCGATGCCTGAACGAAAAT & 10 & 1400 & \\
\hline & 4 & $\mathrm{~F}$ & AGCAAGGGGAAGCCTCACTCATTC & 68 & 593 & 124 & & 4 & $\mathrm{~F}$ & $\begin{array}{l}\text { CAGCGAAGGGAAGGCTCACTCATT } \\
\end{array}$ & 60 & 184 & & & 4 & $\mathrm{~F}$ & GACCTTGAGATGGGGAGACAAC & G & 1730 & \\
\hline & & $\mathrm{R}$ & TATGGCCCCTGTGTCTGTCCTCTG & .0 & (1) & $1,2,4$ & & & $\mathrm{R}$ & CCAAGCTCATCTTCCTTACAACCA & 100 & 404 & & & & $\mathrm{R}$ & GACATGTCTGTCTGTGTGTTT & 104 & 17105 & \\
\hline & 5 & $\mathrm{~F}$ & AATGCCTCTTCTCCTCCAGGTCTA & 65 & 391 & 4 & & 5 & $\mathrm{~F}$ & AGAGATAGGGTGGAGGGTGAGACA & 68 & 625 & & 2DL4 & 1-2 & $\mathrm{F}$ & TGGCCGTTGCGCATGATGTGA & 77 & 553 & \\
\hline & & $\mathrm{R}$ & СTCTCCTCTGGGTCTCTCCTGACCG & 60 & TI & 4 & & & $\mathrm{R}$ & TGCCTGGGTTTCTGGAGCCCTAAT & 100 & $0<5$ & & & & $\mathrm{R}$ & TGTATTGGGGTGAAGATGGCAACC & 111 & 500 & \\
\hline & 6 & $\mathrm{~F}$ & TCAAGACAGTGGGCGTCACATACA & 68 & 290 & 4 & & 6 & $\mathrm{~F}$ & TCAAGACAGTGGGCGTCACATACA & 71 & 858 & & & 3 & $\mathrm{~F}$ & GGGAGGGAGGGGCAGCTCAACATA & 68 & 441 & 1 \\
\hline & 0 & $\mathrm{R}$ & TCAATGCCTGCATCGAAGGTTTCT & 00 & 250 & 4 & & 101 & $\mathrm{R}$ & TGAGATGCACTTCATGCTTTGAGC & 111 & 030 & & & 0 & $\mathrm{R}$ & TTGGGGCCTGGATGATCGGACTC & 100 & 441 & 1 \\
\hline & 7 & $\mathrm{~F}$ & AAAGCTGGGTCTCCCTCCATCTGG & 64 & 410 & 24 & & 7 & $F$ & AGGCCCAACCTCCCACAATG & 71 & 535 & 5 & & 5 & $\mathrm{~F}$ & GGCCATAGAGCAGGGCAGTGAGTT & 66 & 4 & \\
\hline & $r_{1}$ & $\mathrm{R}$ & GAAGGCAGGGACAGGGAGTCTGGT & 04 & 410 & 2,4 & & 170 & $\mathrm{R}$ & AGGGGAAGGGAATCTGGTGCTCTC & tit & 530 & 5 & & 5 & $\mathrm{R}$ & CCTGGGTACCCGGAGCCCTTACT & 100 & 004 & \\
\hline & 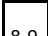 & $\mathrm{F}$ & GAGGGACCTCAGGCTCCTATGG & 70 & 117 & 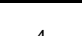 & & 80 & $\mathrm{~F}$ & TCATGGGATGGGTCCTTGAACTC & 68 & 738 & 5 & & 6 & $\mathrm{~F}$ & GTGTGATGCTCCTGTTTTCTCCTT & 10 & 571 & \\
\hline & $\left.\right|^{8-9}$ & $\mathrm{R}$ & ATCAGGGCTCAGCATTTGGAAGTT & 10 & 413 & 4 & & $8^{8-9}$ & $R$ & TGAAATGGAGAATTGTGGGCTAAG & 68 & 738 & 5 & & 6 & $\mathrm{R}$ & CAAGCCCCCAGGCATTTGTCCTC & 68 & $5 / 1$ & \\
\hline 3DL1/S1 & 1 & $\mathrm{~F}$ & ATAGTGAAGGACGCGAGGTGTCAA & 64 & 322 & & 3DL2 & & $\mathrm{F}$ & ATGCAAGGTGGCAATTGTAGTCAC & 68 & 329 & & & 7 & $\mathrm{~F}$ & СACCCCTCCCAATAGGCACAACTC & 77 & 495 & \\
\hline & & $\mathrm{R}$ & ACCTCCAGGTCCAGATCTCCATCC & & JEL & & & & $\mathrm{R}$ & TCCAGGCCCATATCTTTCCCTCTA & & & & & & $\mathrm{R}$ & TGCCTTGGGCCAGAGACTTTCCTG & TI & 700 & \\
\hline & 2 & $\mathrm{~F}$ & ATCTGGGCCTGGAGGCTCAGTCTC & 64 & 373 & & & 2 & $\mathrm{~F}$ & GCAGGGAGGCTAAGTTTACCTTCA & 68 & 299 & & & $8-9$ & $\mathrm{~F}$ & GCACCTATGGCCTCCCCCTGTGT & 7 & 564 & \\
\hline & & $\mathrm{R}$ & CCGGGGAAGGCAAGGTCAGAAA & 04 & Jis & & & & $\mathrm{R}$ & GTCAGAAATGTGGGCCGAGTATCC & 100 & 295 & & & | & $\mathrm{R}$ & TTGTGGTGTGAGGAAGAGTGATGC & tit & 304 & \\
\hline & 3 & $\mathrm{~F}$ & $\begin{array}{l}\text { GACGCCAAGTCTATGCAGGATGG } \\
\end{array}$ & 71 & 417 & & & 3 & $\mathrm{~F}$ & $\begin{array}{l}\text { CTTAGAAAGCGGAAATGGGAGAAT } \\
\end{array}$ & 60 & 183 & & $2 \mathrm{DS} 5$ & 1 & $\mathrm{~F}$ & GAGGGAGGGACAGAGACAGA & 64 & 17 & \\
\hline & & $\mathrm{R}$ & GACGGACACCCCCACCAGAAGC & (1) & 411 & & & & $\mathrm{R}$ & ATACAGTTGGGGCCTGGGTCGATGG & 100 & 400 & & & & $\mathrm{R}$ & CCTCCCATCCAGGTTCCTAT & 104 & J46 & \\
\hline & 4 & $\mathrm{~F}$ & GGACCCCAAGTTCACACAGCATAC & 66 & 496 & & & 4 & $\mathrm{~F}$ & TGGGCACAGAAAAGACACGGAGAC & 68 & 801 & & & 2 & $\mathrm{~F}$ & CCAAGACTCACAGCCTAGTGGGGA & 60 & 370 & \\
\hline & 4 & $\mathrm{R}$ & GAGGAGAGAGACAGACACGGGGAG & 60 & 490 & & & 4 & $R$ & CCTCGTAGAAGCACTTTGTGGAGA & 100 & 801 & & & 2 & $\mathrm{R}$ & GGATCTGCTGTTCCTACCACTTT & 100 & 370 & \\
\hline & & L1F & CAGGTATGAGGGGAGCTATGACAA & 71 & 403 & & & 5 & $\mathrm{~F}$ & CAGGTATGAGGGGAGCTGTGACAA & 68 & 882 & & & 4 & $\mathrm{~F}$ & GAAGGAGAGAGACAGACACCAGGG & 60 & 640 & \\
\hline & 5 & L1R & CTGCCATCCTGCGCCCTGACTC & $\pi 1$ & 403 & & & 5 & $\mathrm{R}$ & AGCGCCAAGATTACAACCGTGAAC & 68 & 882 & & & 4 & $\mathrm{R}$ & TCTGTCTTTTCTAACTCTCGGAAA & 60 & 640 & \\
\hline & 3 & $S 1 \mathrm{~F}$ & GATTGATGGATAGATAGACAGATAG & 60 & & & & 6 & $\mathrm{~F}$ & CAAGACTCCCAGGGTCCAACATTA & & & & & & $\mathrm{F}$ & GAGAATTTGTAGATAGGCACGGAA & & & \\
\hline & & \begin{tabular}{|l|} 
S1R \\
\end{tabular} & GCCATCCTGCGCCCTGACTC & 60 & 896 & & & 6 & $R$ & CACCTTGATTTTAGCCCAGTGACA & 68 & 830 & & & 5 & $\mathrm{R}$ & GTTGAGGGGCTGAGGGAACC & 60 & 980 & \\
\hline & ( & $\mathrm{F}$ & CCCAAGACTCCCAGGGTCCAACAT & 71 & קڤर & & & 7 & $F$ & CCGCCATCAGGCTGCTTGTCCT & 68 & 521 & & & & $\mathrm{~F}$ & GTCTCAGCTCCATAAAATGAGGG & 00 & 60 & \\
\hline & 6 & $\mathrm{R}$ & GCTGGGAGGTTTGAGCCAACACTT & 71 & 202 & & & & $\mathrm{R}$ & CCCCATCCCATGAAGTGCTCTCAA & 68 & 534 & & & 6 & $\mathrm{R}$ & GAGAGTGTTGGCCATGAACCATC & 60 & 360 & \\
\hline & 7 & $\mathrm{~F}$ & TGGGTGCTTGTCCGAAAGAGATGC & 64 & 391 & & & 8 & $\mathrm{~F}$ & CAGCCTCCCCCTGTGGGTTGGT & 77 & 214 & & & 7 & $\mathrm{~F}$ & CCATGTGAGGTTTGAAGGGGTCAG & 60 & 500 & \\
\hline & & $\mathrm{R}$ & CTGAAGGCAGGGGCAGGGAGTCT & 64 & 391 & & & 8 & $\mathrm{R}$ & GAGGGTGCTCACATTTTTCAGGAC & 171 & 214 & & & & $\mathrm{R}$ & AGGGGAAGGGAATCTGGTGCTCTC & 100 & 500 & \\
\hline & & L1F & AGCTCTTTTGTTGACTTCCGTCTC & 68 & 514 & & & 9 & $\mathrm{~F}$ & CTCGGCCCAGCCTCACGGATAC & 66 & 456 & & & $8-9$ & $\mathrm{~F}$ & TTTGAGGAAGAGCGATCCCCTAAG & 77 & 9२० & \\
\hline & 80 & L1 R & GTGAGGAGGAGCGATGCCCTAAGA & $\infty$ & 114 & & & & $\mathrm{R}$ & AGTGGCGACCTTAGGCATTTGTAA & 100 & 400 & & & | & $\mathrm{R}$ & CATGTGGGGAAGCAGGATGGTA & 171 & $5<0$ & \\
\hline
\end{tabular}




\section{Titles and Legends to Figures}

\section{Figure 1}

Patient KIR haplotypes. Genes are ordered according to Hsu et al. (21). Alleles are designated based on the sequences in the KIR Sequence database. Patient MoGe2-2 is of German origin; MoLb1-4 is Lebanese and MoPa61-3 Pakistani. Genbank numbers for subjects with previously identified alleles DQ371509-1645.

\section{Figure 2}

SNPs identifying new alleles for KIR2DL1 and KIR3DL3. Nucleotide positions are based on IPD-KIR sequence alignment database, release 1.1.1, 11 October 2005 (http://www.ebi.ac.uk/ipd/kir/align.html). a) Patient SNPs in KIR2DL1 as compared to the reference allele, KIR2DL1*001. Patients MoLb1-4 and MoPa61-3 have SNPs corresponding to alleles *002 and *00302, respectively, whereas MoGe2-2 has a combination of SNPs not found in any reported allele. b) Patient SNPs in KIR3DL3 as compared to the reference allele KIR3DL3 *001. Patient MoGe2-2 has SNPs corresponding to allele *003. Patients MoLb1-4 and MoPa61-3 do not correspond to any known allele. The SNPs in MoLb1-4 that are not found in the database are indicated in bold.

\section{Table 1}

Primers and conditions used to amplify KIR genes. Primers were designed using Primer Select v5.05 (DNASTAR). Reactions consisted of $60 \mathrm{ng}$ DNA template, 1x PCR buffer (Qiagen), $100 \mu \mathrm{M}$ dNTP, $0.48 \mu \mathrm{M}$ each primer, and 1 unit Taq polymerase (Qiagen) in a 
$50 \mu \mathrm{l}$ final volume. Where indicated 1x Q Solution (Qiagen) was included in the reaction mix. Cycling conditions for all primers were: $94^{\circ} \mathrm{C}$ x 4 min, then 35 cycles $\left(94^{\circ} \times 45 \mathrm{~s}, 45\right.$ $\mathrm{s}$ at temperature indicated in table $1,72^{\circ} \mathrm{C} \times 45 \mathrm{~s}$ ) and $72^{\circ} \mathrm{C} \times 5 \mathrm{~min}$ (Robocycler, Stratagene). Products were visualised on 1\% agarose gels stained with ethidium bromide. In cases where it was not possible to design unique primers, the PCR product was extracted from the gel using the QIAEX II gel extraction kit (Qiagen) and cloned using the TOPO TA cloning kit (Invitrogen). Positive colonies where reamplified by PCR using the same initial primers and conditions and the PCR product sequenced. All sequencing was performed at Genome Quebec using a 3730XL DNA Analysis System (Applied Biosystems). Sequences were analysed using Seqman software v5.05 (DNASTAR). The exons are numbered according to the domain they encode in order to preserve the exon structure homology between the genes. These primers have also been used to amplify these genes in heterozygous subjects (data not shown) and were equally effective. Notes: ${ }^{1}$ primers worked using Q Solution from Qiagen. ${ }^{2}$ amplicons cloned prior to sequencing. ${ }^{3}$ primers that amplify both $K I R 2 D L 1$ and KIR2DS1 if present in the same individual. ${ }^{4}$ primers that amplify both KIR2DL3 and KIR2DL2 if both genes are present in the same individual. ${ }^{5}$ primers that amplify KIR2DS4 and KIR2DS1 if present in the same individual. 